\title{
Gingival Depigmentation: A Surgery for Aesthetics
}

\author{
Dr. Ajit Kumar Yadav, ${ }^{1}$ Dr. Dhirendra Kumar Giri ${ }^{1}$ \\ ${ }^{1}$ Department of Periodontology and Oral Implantology, Nobel Medical College, Biratnagar, Nepal.
}

\begin{abstract}
Smile expresses a feeling of joy, success, sensuality, affection, and courtesy, and reveals self-confidence and kindness. The harmony of the smile is determined not only by the shape, the position, and the colour of the teeth, but also by the gingival tissues. Although melanin pigmentation of the gingiva is completely benign and does not present a medical problem, complaints of "black gums" are common, particularly in patients having a very high smile line. Thus, perio-aesthetic treatment modalities strive to achieve a harmonious interrelationship of the pink with white, which is imperative of all treatment procedures. Excessive gingival pigmentation is a major aesthetic concern for many people complaining of dark gums as unaesthetic. Aesthetic gingival depigmentation can be performed in such patients with excellent results. A case is reported here in which a simple and effective surgical depigmentation was performed without the use of any sophisticated instruments or apparatus.

Keywords: Depigmentation; gingiva; melanin; physiologic pigmentation; scalpel surgery.
\end{abstract}

\section{INTRODUCTION}

Melanin hyper-pigmentation of gingiva usually does not present as a medical problem, but many patients may consider their black gums to be unaesthetic. ${ }^{1}$ This problem is aggravated in patients with a "gummy smile." It is known to be caused by melanin granules within the gingival epithelium and occurs in all races. It is the result of melanin granules produced by melanoblasts intertwined between epithelial cells at the basal layer of gingival epithelium. It is seen as a genetic trait in some populations irrespective of age and gender hence termed as physiologic or racial gingival pigmentation. The degree of pigmentation varies from one individual to another which is mainly dictated by the melanoblastic activity. ${ }^{2}$ The foremost indication for depigmentation therapy is the demand by a person for improved aesthetics.

Demand for cosmetic therapy of gingival hyper-pigmentation is common. Various methods used are gingivectomy (Bergamaschi et al, 1993), gingivectomy with free gingival autografting (Tamizi, 1996), acellular dermal martrix allografts (Pontes et al, 2006), electrosurgery (Gnanaesekhar,

\section{Correspondence:}

Dr. Ajit Kumar Yadav

Department of Periodontology and Oral Implantology,

Nobel Medical College, Biratnagar, Nepal.

e-mail:drajit013@gmail.com

\section{Citation}

Yadav AK, Giri DK. Gingival Depigmentation: A Surgery for Aesthetics. J Nepal Soc Perio Oral Implantol. 2019;3(5):29-31
1998), cryosurgery (Yeh, 1998), abrasion with diamond bur (Bishop, 1994), and various types of lasers (Stabholz et al, 2003). ${ }^{3}$

The present case report describes a simple, effective surgical depigmentation technique providing aesthetically acceptable results and patient's satisfaction.

\section{CASE REPORT}

An 18-year-old female patient visited the department of Periodontology and Oral Implantology, Nobel Medical College and Teaching Hospital, Biratnagar, Nepal, with the chief complaint of blackish gums.

Oral examination revealed pigmented gingiva from right first premolar to left first premolar in maxillary in mandibular region. The patient requested for aesthetically better gums, a scalpel surgery was planned to perform the depigmentation. The entire procedure was explained to the patient and written consent was obtained. To rule out any contraindication for surgery, a complete medical examination, family history and blood investigations were done. Oral prophylaxis was done before the surgery.

Local anaesthesia was infiltrated in the maxilla from premolar to premolar (lignocaine with adrenaline in the ratio 1:2,00,000). A Bard Parker handle with a No. 15 blade and hand piece with diamond bur were used to remove the pigmented layer. After removing the entire pigmented epithelium along with a thin layer of connective tissue with the scalpel, abrasion with diamond bur was done to get the 


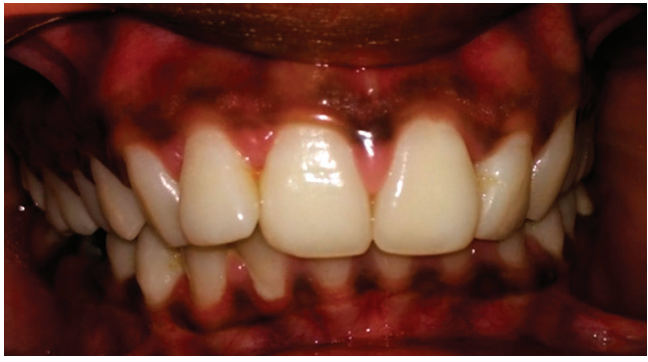

Figure 1: Pre-operative.

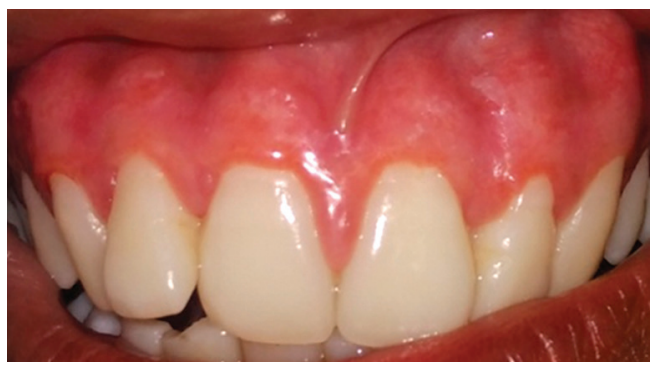

Figure 3: After one week.

physiological contour of the gingiva, the exposed surface was irrigated with saline. Pressure was applied with sterile gauze soaked in local anaesthetic agent to control haemorrhage during the procedure. While using the bur minimal pressure was applied with feather light brushing strokes and without holding bur in one place. Care was taken to see that all remnants of the pigment layer were removed. The surgical area was covered with a periodontal dressing.

Post-surgical Analgesics (ibuprofen with paracetemol, thrice daily for one day then s.o.s) were prescribed. The patient was advised to use chlorhexidine mouthwash twice daily for one week. The patient was reviewed at the end of one week. The healing process was proceeding normally and patient did not report any discomfort. At the end of one month, reepithelialisation was complete and healing was found to be satisfactory. Patient had no complaints of postoperative pain or sensitivity. At the end of six months, the gingiva appeared healthy and no further re-pigmentation was seen.

\section{DISCUSSION}

The intensity and distribution of pigmentation of the oral mucosa is variable, not only between races, but also between different individuals of the same race and within different areas of the same mouth. Physiologic pigmentation is probably genetically determined, but as Dummet $(1960)^{6}$ suggested, the degree of pigmentation is partially related to mechanical, chemical, and physical stimulation. Melanin pigmentation is frequently caused by melanin deposition by active melanocytes located mainly in the basal layer of the oral epithelium. ${ }^{4}$ Pigmentations can be removed for aesthetic reasons. Different treatment modalities (Electrosurgery, Cryosurgery, Lasers, and Scalpel surgery) have been used for

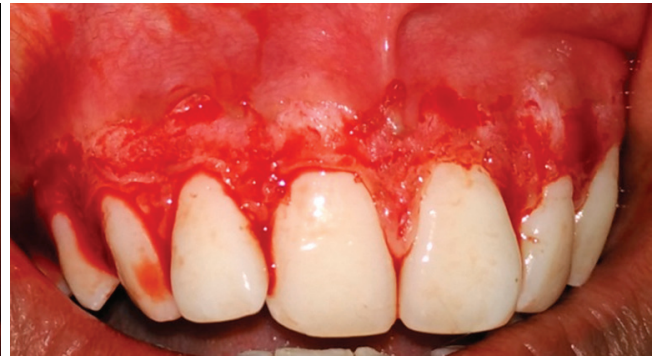

Figure 2: After removal of pigmented layer.

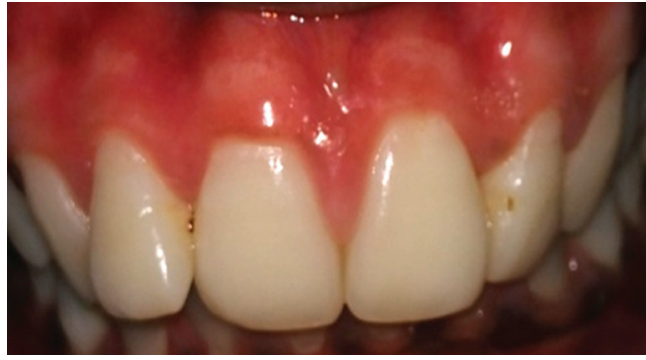

Figure 4: After six months.

this aim (Pontes et al, 2006). ${ }^{3}$ The selection of a technique for depigmentation of the gingiva should be based on clinical experience, patient's affordability and individual preferences.

The surgical removal of undesirable pigmentation using scalpels is one of the first and still popular techniques to be employed. ${ }^{5}$ The procedure essentially involves surgical removal of gingival epithelium along with a layer of the underlying connective tissue and allowing the denuded connective tissue to heal by secondary intention. ${ }^{7}$ The new epithelium that forms is devoid of melanin pigmentation. In this particular case the scalpel method of depigmentation showed better results from both clinical and patients' point of view. The area healed completely in 10 days with normal appearance of gingiva. The use of scalpel technique for depigmentation is the most economical as compared to other techniques, which require more advanced armamentarium. However, scalpel surgery causes unpleasant bleeding during and after the operation, and it is necessary to cover the surgical site with periodontal dressing for 7-10 days.

Post surgical re-pigmentation of gingiva has been previously reported. Re-pigmentation is described as spontaneous and has been attributed to the activity and migration of melanocytic cells from surrounding areas. ${ }^{8}$ The case is being followed up to estimate further the extent and rate of repigmentation.

The depigmentation procedure was successful and the patient was satisfied with the result. Depigmentation of hyperpigmented gingiva by scalpel surgery with bur abrasion is simple, easy to perform, cost effective and above all it causes less discomfort and is aesthetically acceptable to the patient. 


\section{REFERENCES}

1. Almas K, Sadiq W. Surgical Treatment of Melanin-Pigmented Gingiva: An Esthetic Approach. Indian J Dent Res. 2002;13( 2):70-3.

2. Thangavelu A, Elavarasu S, Jayapalan P. Pink esthetics in periodontics - Gingival depigmentation: A case series. J Pharm Bioallied Sci. 2012; 4(Suppl 2):S186-90.

3. Shah C, Dave R, Shah M, Dave D. Evaluation of Scalpel versus Diode Laser for Gingival depigmentation: A Case Report. Int J Adv Health Sci. 2014;1(2):24-7.

4. Cicek Y. The Normal and Pathological Pigmentation of Oral Mucous Membrane: A Review. J Contemp Dent Prac. 2003;4(3):76-86.

5. Cobb CM. Lasers in Periodontics: A Review of the Literature. J Periodontol. 2006;77:545-64.

6. Dummet CO. First symposium on oral pigmentation. J Periodontol. 1960;31(5):345-85.

7. Gnanaesekhar JD, Al-Duwairi YS. Electrosurgery in dentistry. Quint Int (Berlin). 1998;29(10):649-54.

8. Mokeem SA. Management of Gingival Hyperpigmentation by Surgical Abrasion: Report of Three Cases. Saudi Dent J. 2006;18( 3):162-6. 